\title{
Influencia de la cultura familiar en la actividad exportadora de la mediana empresa familiar Ecuatoriana
}

\section{Influence of the family culture on the exporting activity of the Ecuadorian family business}

Esteban Andrade-Rodas

Universidad Internacional del Ecuador, Ecuador

Jaime Salomón

Pontificia Universidad Católica del Perú, Perú

Autor para correspondencia: fandrade@uide.edu.ec, jaime.salomon@pucp.edu.pe

Fecha de recepción: 31 de Mayo de 2017 - Fecha de aceptación: 1y de Septiembre de 2017

\section{Resumen}

A pesar que la empresa familiar tiene un rol dominante en la economía mundial, el conocimiento empírico sobre su internacionalización resulta escaso, evidenciándose en la literatura la necesidad de estudiar la internacionalización de la empresa familiar. A partir de una muestra tomada en medianas empresas familiares ecuatorianas, este trabajo investiga la influencia de la cultura familiar en la actividad exportadora. Los hallazgos revelan la existencia de una relación curvilínea entre las variables cultura familiar y actividad exportadora, llegando a determinarse los rangos en las cuales esta influencia puede ser positiva o negativa. Por lo cual, dado el nivel de influencia de la familia en la empresa, en lo respecta a la internacionalización el ser una empresa familiar no presenta mayores ventajas.

Palabras claves: empresa familiar; cultura familiar; actividad exportadora; pequeña y mediana empresa ecuatoriana

\begin{abstract}
Despite that family business plays a role in world wide economy, its internationalization empirical knowledge is limited; therefore, the need of studying this process is evident. Based on a mediumsized-Ecuadorian enterprise sample, the influence of the family culture in export activities is studied in this document. Outcomes on a curvilinear relationship between the mentioned factors have been found to determine the ranges where the influence may be positive or negative. Thus, with a family influence level on the enterprise given, as per the internationalization in a family business does not offer relevant advantages.
\end{abstract}

Key words: family firm; family culture; export activity; medium sized ecuadorian enterprise 


\section{Introducción}

La empresa familiar constituye la forma de propiedad más extendida a nivel mundial y probablemente la más antigua, representando la mayor cantidad de empresas en el mundo, donde la mayoría de ellas está compuesta por pequeñas y medianas empresas, y su contribución a la economía, producción y empleo es relevante, sin que exista información que muestre completamente la propiedad de las empresas en el mundo (Calabrò \& Mussolino, 2013; Kontinen \& Ojala, 2012; Salomón, 2010; Segaro, Larimo, \& Jones, 2014). La internacionalización constituye hoy en día una estrategia necesaria para las empresas, independientemente de su tamaño, estructura de propiedad, país de origen o sector industrial, dado que ésta representa un mecanismo que permite el crecimiento de largo plazo y supervivencia de la empresa, redundando en una mayor competitividad (Calabrò \& Mussolino, 2013; Cerrato \& Piva, 2012). La empresa familiar no constituye un conjunto homogéneo de empresas, pues el ámbito de la familia tiene impacto en la misma, particularmente en la mediana empresa donde los recursos financieros y no financieros son limitados, sumado a problemas culturales y políticos; por lo cual su proceso y estrategia de internacionalización difiere de la empresa no familiar, (Segaro et al., 2014; Swoboda \& Olejnik, 2013).

La literatura evidencia falta de consenso sobre si la naturaleza familiar de la empresa, restringe o impulsa su internacionalización (Merino, Monreal-Pérez, \& Sánchez-Marín, 2012; Mitter, Duller, Feldbauer-Durstmüller, \& Kraus, 2014). El estudio realizado por Segaro et al. (2014) determinó una relación negativa entre la cultura familiar y el grado de internacionalización, sin embargo el estudio realizado por Mitter et al. (2014) determinaron una relación con forma de U-invertida entre la influencia de la familia y la internacionalización, donde las empresas familiares con niveles medios de cultura familiar es mayormente probable que estén activas a nivel internacional. Tanto el estudio realizado por Segaro et al. (2014) como el realizado por Mitter et al. (2014) además de la influencia de la cultura familiar, consideraron otras variables, así en el primer caso incluyeron las variables: orientación a la administración, flexibilidad estratégica y experiencia en la industria del equipo gerencial; en el segundo las variables: generación, presencia de gerentes no miembros de la familia, la existencia de un consejo de supervisión y consejo asesor en la empresa familiar.

El presente estudio busca examinar empíricamente la influencia de la cultura familiar en la actividad exportadora de la mediana empresa familiar ecuatoriana, llegando a determinar el tipo de relación entre las variables citadas. Para cumplir este propósito, el presente estudio es cuantitativo bajo el paradigma positivista, siguiendo un enfoque de investigación deductivo y realizado en un solo momento del tiempo, por lo cual tiene carácter transversal. Utiliza un modelo de ecuaciones estructurales de mínimos cuadrados parciales [partial least squares] (PLSSEM) basado en varianza, mismo que permite analizar relaciones causa efecto entre constructos latentes, siendo una técnica que se encuentra en etapa de temprano desarrollo para el estudio de empresas familiares (Hair, Ringle, \& Sarstedt, 2011; Nitzl, Roldan, \& Cepeda, 2016; Sarstedt, Ringle, \& Hair, 2014; Sarstedt, Ringle, Smith, Reams, \& Hair, 2014). No se han identificado estudios de este tipo en Ecuador, por lo que además se busca aportar con evidencia empírica en un país en vías de desarrollo. El estudio aporta al conocimiento sobre la empresa familiar y particularmente a la internacionalización de la mediana empresa, ayudándola a desarrollar 
capacidades "con el objetivo de incrementar sus probabilidades de supervivencia y continuidad" (Salomón, 2010, p. 6).

\section{Revisión de la Literatura e Hipótesis}

La literatura relacionada a la internacionalización de la empresa familiar ha incrementado su búsqueda sobre el rol de la familia propietaria, los tipos y niveles de propiedad, sin embargo, el rol de una dimensión más suave de propiedad como la cultura organizacional no ha recibido mayor atención (Sciascia, Mazzola, Astrachan, \& Pieper, 2012; Segaro et al., 2014). A través del tiempo, el compromiso de la familia con la mediana empresa familiar desarrolla su cultura organizacional, la cual ejerce una poderosa influencia que se extiende a todos sus miembros y la manera en que ellos interpretan y responden a los cambios externos, e influye en su misión, es decir lo que la familia busca lograr y cómo desea lograrlo (Craig, Dibrell, \& Garrett, 2014; Segaro et al., 2014; Zahra, Hayton, Neubaum, Dibrell, \& Craig, 2008). La cultura familiar hace referencia a los supuestos acerca de la forma en que se debe manejar el negocio y los valores que comparte la familia, otros propietarios, gerentes y empleados (Merino et al., 2012). Representa la fusión de la familia y sus valores organizacionales, incorporando tanto la cultura como el compromiso organizacional y constituye un método para evaluar el grado en el cual los valores de la empresa y la familiar se sobreponen, así como el compromiso e influencia de la familia con la empresa, a través de la medición del impacto de la familia en los resultados, permitiendo diferenciar la empresa familiar de la no familiar (Astrachan, Klein, \& Smyrnios, 2002; Klein, Astrachan, \& Smyrnios, 2005; Mitter et al., 2014; Segaro et al., 2014; Smith, Hair, \& Ferguson, 2014; Zahra et al., 2008). Como resultado de esta fusión familia y empresa, los supuestos y valores no pueden ser fácilmente conferidos a otros países (Mitter et al., 2014).

Diversas teorías y pruebas empíricas determinaron que la influencia de la familia puede tanto facilitar como inhibir la internacionalización de la empresa familiar (Mitter et al., 2014). Muchos factores inhiben la internacionalización de la mediana empresa familiar, de acuerdo a Merino et al. (2012) los principales son: (a) factores estratégicos, relacionados a la falta de recurso y orientación a su mercado doméstico; (b) problemas familiares, relacionados a la falta de preparación o actitud de la familia; y (c) factores individuales, relacionados a la resistencia que surge desde el equipo gerencial compuesto por miembros de la familia. Fernández y Nieto (2005) identificaron adicionalmente factores como: falta relaciones en el extranjero, falta de conocimiento o experiencia en mercados internacionales, y equipos humanos poco preparados para la internacionalización.

Por otro lado, la literatura sobre internacionalización muestra diversas ventajas como son: (a) la reducción de la incertidumbre de los ingresos mediante la disolución del riesgo de inversión en varios países, (b) una mejor gestión del riesgo sistemático y no sistemático al incrementar el número de oportunidades de negocio y de operaciones, y (c) reducción de la dependencia de la empresa de determinados proveedores, distribuidores o clientes (Merino et al., 2012). Estas opciones de reducción de riesgo deberían ser atractivas para la empresa familiar al disminuir el riesgo total frente a empresas que operan solo en sus mercados domésticos, sin embargo, la evidencia empírica muestra resultados contradictorios sobre el perfil del riesgo en la empresa familiar, algunas investigaciones muestran que la empresa familiar es conservadora y otras la muestran propensa al riesgo (Fernández \& Nieto, 2005; Mitter et al., 2014; Naldi, Nordqvist, Sjoberg, \& Wiklund, 2007). De acuerdo a Merino et al. (2012) la pequeña y mediana 
empresa familiar presenta menores niveles de internacionalización que empresas no familiares y éstas tradicionalmente operan en su mercado doméstico, debido a la sustancial y fuerte presencia de la familia en la empresa y su preocupación por preservar el control de la misma, con el propósito de entregarla a generaciones futuras. De ahí que la internacionalización para la empresa familiar sea una espada de doble filo, ya que por un lado permite a la empresa familiar diseminar el riesgo en diferentes segmentos geográficos, pero conlleva también una mayor exposición al riesgo como resultado de una mayor incertidumbre, junto con el temor de perder la riqueza familiar, y el control de la empresa, estimulándola a mantener estabilidad y optar por proyectos tradicionales de bajo riesgo (Merino et al., 2012; Mitter et al., 2014; Sciascia et al., 2012).

Por tanto, la relación entre la influencia de la familia y la internacionalización no está clara y se evidencian efectos positivos y negativos, lo cual sugiere una posible relación no lineal (Mitter et al., 2014; Sciascia et al., 2012; Segaro, 2012). En la mediana empresa familiar es probable que el involucramiento de la familia en la administración dé mayor forma a la cultura familiar, proporcionando mayor cohesión y una visión compartida, sin embargo, un excesivo involucramiento podría causar falta de profesionalismo y la presencia de gerentes no calificados, lo que impediría la influencia de personas ajenas en el gobierno de la empresa, afectando su desempeño e internacionalización y llevándola a mantener estándares de comodidad y conformidad (Eddleston, Kellermanns, \& Zellweger, 2012; Ensley \& Pearson, 2005; Segaro et al., 2014; Zahra, 2012).

De acuerdo a Mitter et al. (2014) cuando se presentan niveles moderados de influencia de la familia por su cultura familiar, los propietarios y gerentes no familiares amplían la base de recursos de la empresa familiar y pueden proporcionar a la empresa de las capacidades gerenciales necesarias para su internacionalización. Por el contrario, una escasa influencia de la cultura familiar está asociada con el ingreso o participación de inversionistas externos a la familia y gerentes no familiares, pudiendo generar conflictos de agencia debido a una mayor fragmentación y la separación de la propiedad y la administración, ocasionando que la internacionalización no se realice. Por tanto, se espera exista un cierto rango de influencia de la cultura familia, donde exista una relación positiva con la internacionalización, por fuera de dicho rango, los aspectos negativos de la influencia de la familia podrían prevalecer, por lo que la empresa familiar resulta menos proclive a internacionalizarse. Con este antecedente, se propone una relación curvilínea, entre la influencia familiar y la internacionalización, permitiendo formular la siguiente hipótesis.

Hipótesis 1. La cultura familiar está relacionada de manera curvilínea con la actividad exportadora.

\section{Método}

Como medio de recolección de datos en el trabajo de campo se usaron encuestas, "siendo un método aceptado para recopilar información con la finalidad de estudiar la relación entre variables" (Pino, 2008, p. 92) y ampliamente utilizado en investigaciones relacionadas a la empresa familiar y su internacionalización (e.g., Calabrò \& Mussolino, 2013; Craig et al., 2014; Kuivalainen, Saarenketo, \& Puumalainen, 2012; Merino et al., 2012; Segaro et al., 2014; Zahra et al., 2008). 
El instrumento aplicado se derivó de estudios previos, por lo cual los indicadores de medida de los constructos se basaron en escalas existentes, por lo cual, de acuerdo a Pino (2008) "cuando se derivan las variables de una revisión de la literatura, la validez de contenido está asegurada" (p. 107). Las preguntas utilizadas para evaluar la variable cultura familiar fue aplicado por Zahra et al. (2008) en un estudio cuantitativo realizado con empresas familiares en los Estados Unidos y posteriormente por Segaro et al. (2014) en un estudio en pequeñas y medianas empresas familiares en Finlandia, mismos que utilizaron la escala de influencia de la familia (Escala F-PEC) que fuera desarrollada por Astrachan et al. (2002) y está formada por tres dimensiones: poder $(\mathrm{P})$, experiencia $(\mathrm{E})$ y cultura $(\mathrm{C})$. Esta escala ha sido utilizada y validada en numerosos estudios, entre ellos Klein et al. (2005) realizaron rigurosas pruebas concluyendo que la escala usada en la medición demostró altos niveles de confiabilidad. Rutherford, Kuratko, y Holt (2008) utilizaron la escala F-PEC para medir la relación entre la familiaridad [familiness] y el desempeño empresarial, así mismo Mitter et al. (2014) utilizó esta escala para medir la relación entre la influencia de la familia y la internacionalización de la empresa. La variable cultura familiar se valoró usando una escala de Likert de cinco puntos, desde 1: no, en lo absoluto, hasta 5: totalmente en gran medida.

La internacionalización se evaluó por la actividad exportadora de la empresa familiar, la cual proporciona una medida instantánea del desarrollo de la empresa a través del tiempo (Kuivalainen, Sundqvist, Saarenketo, \& McNaughton, 2012). La actividad exportadora como variable endógena se midió usando el criterio propuesto por Merino et al. (2012) Que consideraron en primer lugar la propensión exportadora de la empresa, misma que identifica si la empresa exportó durante el período considerado en el estudio, y en segundo lugar la intensidad exportadora, como indicador, el cual fue medido por el porcentaje de las ventas en mercados externos respecto de las ventas totales de la empresa, criterio que ha sido utilizado en números estudios (e.g., Graves \& Thomas, 2006, 2008; Riahi-Belkaoui, 1998).

El cuestionario utilizado estaba en idioma inglés, por lo cual se tradujo al idioma español asegurando la claridad e interpretación de cada pregunta. Una vez traducido el instrumento se aplicó en español como prueba preliminar a dos empresas parte de la población y tres académicos conocedores del tema, luego de lo cual, se realizó una entrevista para recoger sus observaciones y basados en esta retroalimentación, ajustar y afinar el cuestionario antes de ser aplicado de manera general. Las preguntas aplicadas constan en la Tabla A1.

\section{Población y Muestra}

La población objeto de estudio está conformada por medianas empresas familiares domiciliadas en Ecuador que se encuentren en operación, para lo cual, las empresas se identificaron considerando: (a) que sean medianas empresas, mismas que, acuerdo a la Decisión 702 y Resolución 1260 de la Secretaría General de la Comunidad Andina, "comprende a todas las empresas formales legalmente constituidas y/o registradas ante las autoridades competentes, que lleven registros contables y/o aporten a la seguridad social" (Comunidad Andina, 2008, p. 2) incluidas dentro de los umbrales definidos tanto del número de empleados (desde 50 hasta 199), como del valor bruto en ventas anuales (desde 1 millón hasta 5 millones) y donde prevalece el valor bruto de ventas anuales (Comunidad Andina, 2008, 2009); (b) tienen carácter familiar, mismo que considera empresas en las cuales la familia posee la mayor cantidad de acciones, 
ejerce su control administrativo y además la empresa se considera así misma como una empresa familiar (Calabrò \& Mussolino, 2013; Graves \& Thomas, 2008; Segaro et al., 2014; Swoboda \& Olejnik, 2013); (c) es productora de bienes, entendiéndose por productora de bienes "aquellas empresas cuya actividad principal se relaciona con la elaboración de bienes tangibles, mientras que en servicios se incluyen todas las demás" (Pino, 2008, pp. 108-109), (d) corresponde al sector privado, por "pertenecer a un agente económico que no está relacionado con el Estado" (Pino, 2008, p. 98); (e) se encuentra en operación, (f) llevan registros contables y (g) realiza actividades de exportación.

De acuerdo al Instituto Nacional de Estadísticas y Censos, entidad pública responsable ante las autoridades nacionales y la Secretaría General de la Comunidad Andina de proveer información estadística del Ecuador, existen un total de 810,272 empresas registradas en el Ecuador, de las cuales 343 pueden ser consideradas medianas empresas, están activas, producen bienes, llevan registros contables, pertenecen al sector privado y realizan actividades de exportación (INEC, 2013). La población no pudo ser completamente definida dado que no existe una base de datos que identifique en Ecuador las empresas como familiares o no, aunque se puede asumir que la mayoría de las medianas empresas lo son (Hiebl, 2013; Kontinen \& Ojala, 2012) por ello, se utilizó una muestra de conveniencia. Investigaciones previas relacionadas con el presente estudio que utilizaron encuestas no se desarrollaron con una muestra probabilística (e.g., Berent-Braun \& Uhlaner, 2010; Calabrò \& Mussolino, 2013; Miller, Le Breton-Miller, \& Scholnick, 2008; Segaro et al., 2014; Zahra et al., 2008). De acuerdo a Miller et al. (2008) la tasa de respuesta que normalmente se obtienen de investigaciones que utilizan encuestas fluctúan entre el $25 \%$ al $35 \%$.

Por conveniencia en la toma de información de campo se ha considerado para el presente estudio las empresas de los sectores florícolas y agro industrial por ser predominantemente exportadoras, mismas que se encuentran localizadas en diferentes provincias ecuatorianas. (INEC, 2014).

\section{Recolección y Análisis de Datos}

La recolección de datos se realizó a través de encuestas con la participación directa de los investigadores. La unidad de análisis es la empresa familiar y se identificaron las empresas de acuerdo a la población determinada, para proceder a contactarlas y solicitar completen el cuestionario. En los casos que alguna de las empresas solicitó completar el formulario de manera electrónica, éste fue preparado en formato digital para permitir que sean llenados directamente, facilitando y reforzando su recolección. Para empresas que no completaron el cuestionario se ofreció facilidades para hacerlo por medio de una conversación telefónica.

Se solicitó que un funcionario por empresa complete el cuestionario, quien debía ser el responsable de su internacionalización, y conocer sobre sus planes y resultados. A los participantes se les indicó respondieran todas las preguntas de forma independiente y evitaran contestar sobre la situación futura, considerando únicamente la situación actual de la empresa. Se les solicitó el respectivo consentimiento, y se aseguró el anonimato y confidencialidad de las respuestas. Para garantizar confidencialidad, no se llevó un registro individual de las empresas o personas que participaron o no en la encuesta, manteniendo así su identidad de manera confidencial. Los resultados únicamente muestran información agregada y no respuestas 
específicas o individuales de cada cuestionario. Con este mecanismo se buscó alcanzar una tasa de participación elevada, y homogeneidad en las personas que respondieron la encuesta.

Inicialmente los datos fueron analizados para verificar que los cuestionarios completados cumplan con lo definido en la población, esperando así disponer de datos que se ajusten a lo propuesto en el estudio. Posteriormente usando el software SPSS® versión 21.0 se exploraron los datos dentro del campo de investigación, se analizaron datos perdidos, valores extremos, casos atípicos, normalidad de datos y se determinó si los datos de los sectores considerados en el estudio, mismos que constituyen muestras independientes, provienen de una misma población. En una siguiente instancia se usó el software WarpPLS versión 5.0 para obtener los resultados del modelo estructural propuesto.

\section{Resultados}

El presente estudio considera empresas de los sectores florícolas y agro industrial, por lo cual se procedió a contactar las mismas a partir de la base de datos facilitada por el Instituto de Promoción y Exportaciones e Inversiones del Ecuador (PRO ECUADOR). Del sector florícola se recolectaron un total de 114 cuestionarios y del sector agro industrial un total de 127 cuestionarios, sin embargo, luego de revisar los formularios recibidos y verificar si éstos se ajustan a la población objeto de estudio, si estaban correctamente llenados y completos, se aceptaron 75 casos para empresas del sector florícola y 42 casos para empresas del sector agro industrial, lo cual representa un total de 117 casos válidos, mismos que respecto a la población constituyen un $34.1 \%$, porcentaje considerado satisfactorio dentro de investigaciones de esta naturaleza. Los resultados de las respuestas del campo de investigación para cada uno de los indicadores se muestran en la Tabla A1 y Tabla A2.

Con 117 casos aceptados como válidos se realizaron diversas pruebas estadísticas con el propósito de explorar la calidad de los mismos. En primer lugar, se analizaron los datos perdidos. En la Tabla B1 se muestra el estadístico de observaciones en el que constan el número de casos válidos, valores mínimos, máximos, media, desviación estándar, asimetría y curtosis de cada indicador reflectivo, así como la cantidad y porcentaje de casos con datos perdidos. Las variables reflectivas FC2, FC8 y FC9 presentan datos perdidos en porcentajes del 1,71\%, 1.71\% y 0.85\% respectivamente, dando un total de 112 casos válidos, lo cual representa un $95.73 \%$ del total de datos. De acuerdo a Kock (2015) como regla general es aceptable que el conjunto de datos no tenga ninguna variable con más del $10 \%$ de sus valores perdidos, una regla más relajada establece un umbral del 20\%. En la presente investigación los datos perdidos se completaron usando el método de imputación aritmético de valores medios [arithmetic mean imputation].

En segunda instancia se buscó detectar casos con valores extremos, cuyo objetivo es "encontrar inconsistencias en el llenado de la encuesta por parte de los participantes" (Pino, 2008, p. 133). El indicador FC1 de la variable cultura familiar, reportó cuatro casos con al menos un dato con valores extremos bajos en una o más preguntas (casos número 72, 74, 108, 114), por lo que éstos fueron analizados sin que se encuentre patrones de respuesta o motivos por los cuales puedan considerarse mal llenados, manteniéndose como válidos y considerándose la totalidad en el análisis. Adicionalmente, se realizó el procedimiento de detección de anomalías por medio de la identificación de casos atípicos, incluyendo en el mismo la totalidad de los 
indicadores del modelo con el propósito de auditar los datos de manera previa a su análisis. El resultado obtenido indicó la no presencia de anomalías.

En tercer lugar, se realizó la prueba de normalidad con el objetivo de establecer si los datos siguen o no una distribución normal. En la Tabla B2 se presentan los resultados obtenidos por el desarrollo de las pruebas de Kolmogorov-Smirnov, Shapiro-Wilk con un intervalo de confianza del $95 \%$ y donde éstos muestran en todas las variables niveles de significancia menores a .05, por lo cual se puede concluir que los datos no siguen una distribución normal. Una de las características del modelo PLS-SEM, es su capacidad de realizar estimaciones de modelos muy complejos sin tener que asumir supuestos sobre la distribución de los datos, característica que no es aceptada por el modelo de ecuaciones estructurales basado en covarianza (Hair, Sarstedt, Pieper, \& Ringle, 2012; Sarstedt, Ringle, \& Hair, 2014; Sarstedt, Ringle, Henseler, \& Hair, 2014). Este resultado ratifica la necesidad de utilizar el método PLS-SEM para la presente investigación.

Finalmente, para determinar si los datos recopilados de los sectores florícola y agro industrial como muestras independientes provienen de poblaciones equivalentes, se desarrolló una prueba de diferencia de medias, aplicando la prueba U de Mann-Whitney y W de Wilcoxon con un nivel de significancia de .05, misma que es una prueba no paramétrica alternativa a la prueba $t$ de dos muestras y por tanto no "requiere suponer que las dos poblaciones sigan la distribución normal y tengan varianzas poblacionales iguales" (Lind, Marchal, \& Wathen, 2008, p. 695). Los resultados se muestran en la Tabla B3, mismos que de acuerdo a los valores del nivel crítico bilateral o significancia asintótica bilateral, son todos superiores a .05 , por lo que se puede concluir que los resultados de las variables correspondientes a los grupos definidos por la variable sector no presentan una diferencia significativa entre sus medias. Con los resultados obtenidos los datos fueron integrados.

Una de las características PLS-SEM es su capacidad de trabajar con tamaños de muestra pequeños, ofreciendo capacidades de muestreo que no brindan otras herramientas de análisis multivariante, mostrándose robusto y relativamente con una alta capacidad estadística, sin que esto signifique que sea un método exento de un adecuado tamaño de muestra (Hair, Ringle, \& Sarstedt, 2013; Sarstedt, Ringle, \& Hair, 2014; Sarstedt, Ringle, Smith, et al., 2014). Hair et al. (2012) recomendaron utilizar la regla de las diez veces [ten time rules] como mecanismo de estimación aproximada para determinar el tamaño de la muestra. Esta regla, para modelos con indicadores reflectivos es equivalente a utilizar un tamaño de muestra diez veces el mayor número de rutas o caminos estructurales que se dirigen a un constructo latente particular en el modelo estructural o modelo interior (Hair et al., 2012; Sarstedt, Ringle, Smith, et al., 2014). Sarstedt, Ringle, y Hair (2014) recomendaron que el mínimo número de observaciones es un rango entre 30 y 100 casos. El tamaño obtenido de muestra en el presente estudio es 117 casos, mismo que supera lo indicado, considerándose por tanto adecuado.

El método PLS-SEM requiere completar dos instancias: (a) evaluar el modelo de medida [measurement model], y (b) evaluar el modelo estructural [structural model] (Hair et al., 2011; Sarstedt, Ringle, Smith, et al., 2014). Para evaluar el modelo de medida, se consideró que éste considera únicamente constructos de medida reflectivos, por lo cual, para establecer su confiabilidad y validez, se analizaron los resultados de: (a) indicador de confiabilidad [indicator 
reliability] llamado también indicador de carga [indicator loadings], (b) confiabilidad de la consistencia interna [internal consistency reliability] del constructo, mismo que se puede lograr evaluando la confiabilidad compuesta [composite reliability]; (c) validez convergente [convergent validity], y (d) validez discriminante [discriminant validity] del modelo de medida (Sarstedt, Ringle, Smith, et al., 2014).

El indicador de confiabilidad se obtiene a partir de la tabla de cargas combinadas y cargas transversales [combined loadings and cross-loadings]. Los valores de carga de las variables latentes reflectivas de acuerdo a Hair et al. (2011) deben ser mayores o iguales a 0.4, valores menores deben ser retirados del modelo y los valores de carga transversal deben ser menores (Kock, 2015). El nivel de significancia (p-value) para todos los indicadores reflectivos se considera un parámetro de validación del análisis factorial confirmatorio, esperando valores menores a .05 (Kock, 2015). De acuerdo a los criterios citados, se retiró el indicador FC8, dado que su valor de carga fue menor al umbral permitido (0.364) a pesar que su nivel significancia fue aceptable $(\mathrm{p}<.05)$. En la Tabla 1 se muestran los indicadores de carga combinada y carga transversal, donde los resultados cumplen con lo especificado.

Tabla 1. Indicadores de Carga Combinada y Carga Transversal

\begin{tabular}{lccccc}
\hline & & Actividad Exportadora & Cultura Familiar & Tipo Indicador & P-Value \\
\hline Actividad Exportadora & FSTS & $(1.000)$ & 0.000 & Reflectivo & $<.001$ \\
Cultura Familiar & FC1 & -0.118 & $(0.552)$ & Reflectivo & $<.001$ \\
& FC2 & -0.149 & $(0.625)$ & Reflectivo & $<.001$ \\
& FC3 & 0.179 & $(0.578)$ & Reflectivo & $<.001$ \\
& FC4 & 0.049 & $(0.718)$ & Reflectivo & $<.001$ \\
& FC5 & 0.099 & $(0.699)$ & Reflectivo & $<.001$ \\
& FC6 & 0.080 & $(0.770)$ & Reflectivo & $<.001$ \\
& FC7 & 0.075 & $(0.762)$ & Reflectivo & $<.001$ \\
& FC9 & 0.064 & $(0.659)$ & Reflectivo & $<.001$ \\
\hline
\end{tabular}

La confiabilidad de la consistencia interna del constructo, se evalúo con la confiabilidad compuesta [composite reliability] y el Alpha de Cronbach (Sarstedt, Ringle, Smith, et al., 2014). De acuerdo a Kock (2015) el resultado de estos indicadores deberían ser igual o mayor a 0.7 para considerarse aceptables. Para la variable cultura familiar el índice de confiabilidad compuesta reportó un resultado de 0.868 y de 0.835 para el Alpha de Cronbach. Estos indicadores reportaron el valor de 1.000 para la variable actividad exportadora.

La validez convergente se evalúo por medio de la varianza media extraída [average variance extracted] (AVE) para todos los ítems asociados con cada constructo (Sarstedt, Ringle, Smith, et al., 2014). El resultado de AVE para la variable cultura familiar fue de 0.524 y de 1.000 para la variable actividad exportadora, lo que indica que en promedio, el constructo explica sobre el 50\% de la varianza de sus ítems (Sarstedt, Ringle, Smith, et al., 2014), evidenciándose la validez convergente del modelo de medida. El resultado de AVE es igualmente usado para evaluar la validez discriminante y cuyos resultados se usan conjuntamente con la correlación de las variables latentes (Kock, 2015).

En la Tabla 2 se presentan los resultados de la correlación entre las variables latentes con las raíces cuadradas de la varianza media extraída [correlations among latent variables with square roots of AVEs]. De acuerdo a Kock (2015) la validez discriminante se evalúa 
considerando que para cada variable latente, la raíz cuadrada de la varianza media extraída debería ser mayor que cualquier otra de las correlaciones que implica las variables latentes. Esto es, los valores de la diagonal de la tabla deben ser mayores que cualquier otro valor por encima o por debajo de ellos en la misma columna, o deben ser superiores a cualquier otro valor de su izquierda o derecha en la misma fila. Como se aprecia, los resultados cumplen con lo especificado logrando el modelo de medida validez discriminante.

Tabla 2. Correlación entre Variables Latentes con las Raíces Cuadradas de AVEs

\begin{tabular}{lcc}
\hline & Actividad Exportadora & Cultura Familiar \\
\hline Actividad Exportadora & $(1.000)$ & -0.189 \\
Cultura Familiar & $-0.189^{\mathrm{a}}$ & $(0.675)$ \\
\hline \multicolumn{2}{c}{ Nota. a. p-value para la correlación $(\mathrm{p}=.042)$}
\end{tabular}

Los criterios para evaluar el modelo estructural consideraron: (a) evaluación del modelo estructural de potencial colinealidad entre los constructos predictores, (b) evaluación del coeficiente de determinación (R2) como indicador de la capacidad predictiva del modelo, (c) evaluación del índice de redundancia de validez cruzada [cross-validated redundancy] (Q2), el cual permite evaluar la relevancia o capacidad predictiva del modelo; y (d) la significancia y relevancia de los coeficientes de ruta [path coefficients]. La colinealidad en general se evaluó por el índice de colinealidad completa o también llamado multicolinealidad de los factores de inflación de la varianza [full collinearity variance inflation factors] (VIFs) (Kock, 2015). El resultado obtenido de VIFs fue de 1.037, lo que no se sugiere la existencia de multicolinealidad en el modelo, dado que como regla general se considera ideal un resultado menor o igual a 3.3. En la Tabla B4 se muestra la correlación entre los indicadores reflectivos, mismos que no presentan valores absolutos de correlación mayores a 0.70 por lo que ninguno de los indicadores está fuertemente correlacionado con los demás (Lind et al., 2008).

El coeficiente de determinación (R2) reportó un valor de 0.065 y el índice de redundancia de validez cruzada (Q2) de 0.066. De acuerdo a Kock (2015) valores de R2 menores a 0.02 sugieren efectos combinados de predictores en el bloque de variables latentes muy débiles para ser relevantes desde el punto de vista práctico y valores de Q2 mayores que cero representa que la exactitud predictiva del modelo es aceptable para dicho constructo en particular (Hair et al., 2011; Sarstedt, Ringle, Smith, et al., 2014). Hair et al. (2013) destacaron la necesidad de incluir el resultado del coeficiente de tamaños de efecto [effect sizes] (f2) para los coeficientes de ruta y cuyo resultado fue de 0.065 . Kock (2015) indicó que valores menores a 0.02 podría representar enlaces o vínculos entre variables despreciables para ser considerados relevantes desde el punto de vista práctico, aun cuando los resultados puedan ser estadísticamente significativos (Hair et al., 2012), por todo lo indicado, el modelo ofrece capacidad predictiva. El resultado del coeficiente de ruta $(\beta)$ fue de -0.254 y presenta significancia estadística $(p=.002)$, por lo cual en primera instancia se puede considerar la existencia de una relación negativa entre la cultura familiar y la actividad exportadora. Finalmente, para determinar con precisión la forma de la relación entre las variables objeto de estudio, se obtuvieron las figuras que muestran el mejor ajuste para la relación bivariante, con datos y sin datos, con escala no estandarizada entre las variables latentes cultura familiar y actividad exportadora. El resultado se presenta en la Figura 1 y para obtener el resultado de la relación se usó el algoritmo Warp3, mismo que trata de identificar las relaciones entre las variables latentes definidas por funciones cuyo primer origen son curvas en forma de U (Kock, 2015). 

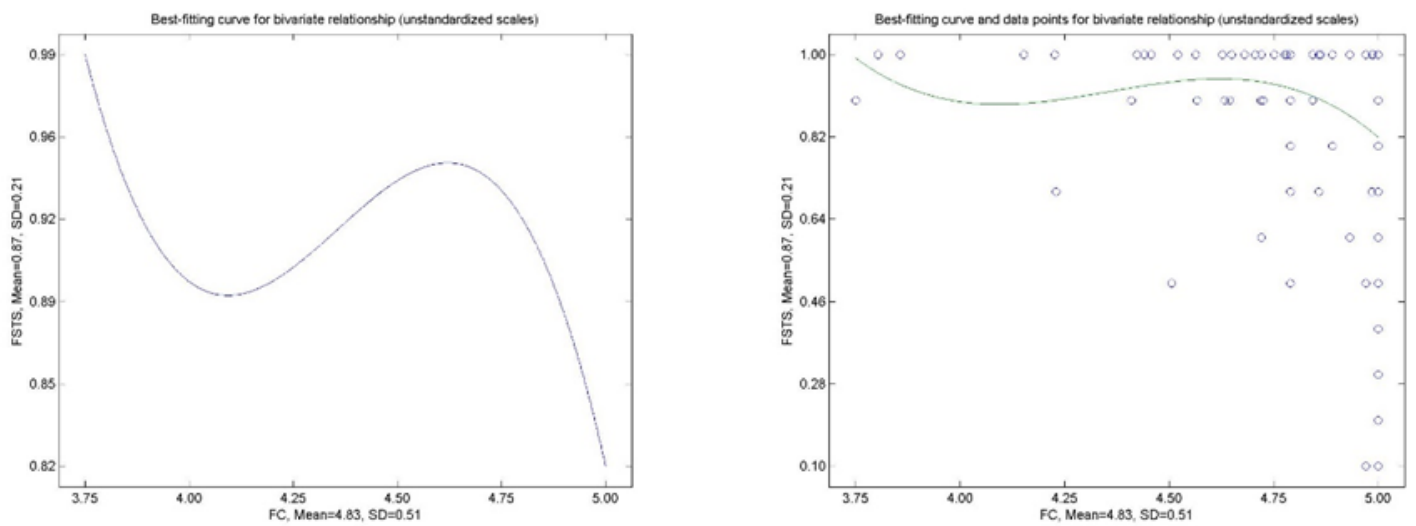

Figura 1. Mejor ajuste de curva sin puntos y con puntos de datos para la relación bivariante con escala no estandarizada entre las variables latentes cultura familiar (FC) y actividad exportadora (FSTS).

Con el resultado obtenido, se puede apreciar que existe una relación curvilínea entre las variables objeto de estudio, por lo cual la Hipótesis 1 es aceptada.

\section{Discusión}

Los resultados obtenidos permiten aceptar la Hipótesis 1, respecto a la relación entre la cultura familiar y la actividad exportadora, al mostrar una relación curvilínea entre las mismas. Por tanto, cuando la influencia de la familia presenta valores de bajos a medio altos y altos, la empresa familiar tiende a mantener su estancia en el mercado local, evidenciando una relación negativa hacia la actividad exportadora. El resultado obtenido del coeficiente de ruta $(\beta)$ fue negativo y estadísticamente significativo, lo cual puede comprenderse, dado que el valor medio obtenido para la variable FC fue de 4.83 con una desviación estándar de 0.51. En la Tabla B4 se presenta la correlación para los indicadores reflectivos entre la cultura familiar y actividad exportadora, para todos ellos es negativa.

En la Figura 2 se presente la curva y segmentos obtenidos para la relación bivariantes, tanto sin escala estandarizada como con escala estandarizada. En la misma, se puede observar que la relación presenta la forma de una $U$ y posteriormente se convierte en una U-invertida. La relación entre la cultura familiar y la actividad exportadora es positiva en el rango comprendido entre 4.15 y 4.56 de la variable cultura familiar (FC), fuera de este rango, la relación es negativa. La literatura evidencia resultados contradictorios en la relación de estas variables, lo cual puede comprenderse a partir del resultado obtenido.
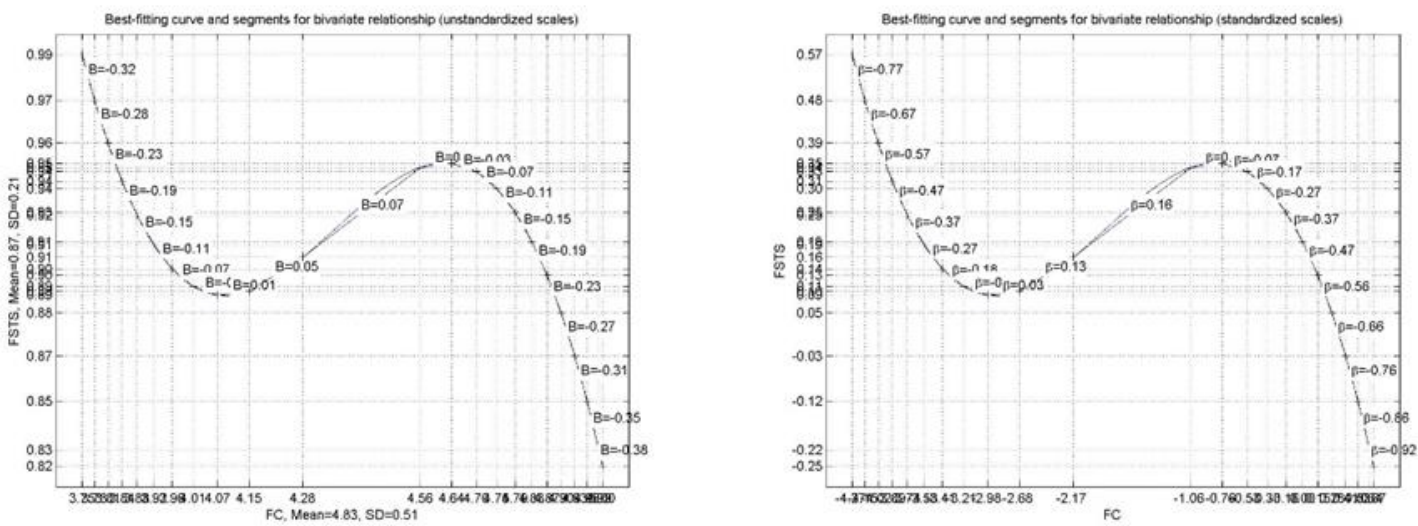
Figura 2. Mejor ajuste de curva y segmentos para la relación bivariante sin escala estandarizada y con escala estandarizada entre las variables latentes cultura familiar (FC) y actividad exportadora (FSTS).

El estudio realizado por Segaro (2012) predijo una relación en forma de una U-invertida para esta relación. Posteriormente Sciascia et al. (2012) confirmaron empíricamente la relación en forma de U-invertida entre la propiedad de la empresa e internacionalización. El estudio realizado Segaro et al. (2014) demostró empíricamente una relación negativa entre la cultura familiar y el grado de internacionalización, luego Mitter et al. (2014) igualmente de manera empírica, demostraron una relación en forma de U-invertida considerando la cultura familiar e internacionalización. En todos estos estudios se consideran variables adicionales o se usaron diferentes métodos.

\section{Conclusiones}

Los sistemas de la familia y la empresa comparten supuestos y valores, e inciden en la cultura, estrategia e internacionalización de la empresa familiar, siendo la cultura familiar la variable que permite evaluar el nivel de involucramiento y compromiso de la familia en la propiedad y administración de la empresa. Las variables cultura familiar y actividad exportadora muestran como resultado una relación curvilínea, mismo que puede comprenderse por la fuerte cultura de la familia presente en la mediana empresa familiar, la cual se desarrolla a lo largo del tiempo, llevándola predominantemente a mantener estándares y conformidad, constituyendo un factor que puede tanto facilitar como inhibir la internacionalización. Por tanto, niveles altos de cultura familiar o niveles de medios a bajos, la empresa tiene mayor orientación a su mercado doméstico y menor propensión a internacionalizarse, mostrándola como un ente cohesionado y adverso al riesgo, fundamentalmente por el temor de la familia a perder la propiedad, el control e incluso sus valores, llevándola a evitar la influencia de personas ajenas a la empresa.

El presente estudio evidencia la vinculación socio-emocional de los miembros de la familia propietaria que participan en la administración de la empresa, el cual podría ser muy alto dado que ésta puede servir para satisfacer las necesidades de seguridad, contribución social, aceptación y reputación de la familia. Esto lleva a la empresa familiar a orientarse a su mercado local como un mecanismo para evitar perder dicha vinculación, identificando a la cultura familiar como uno de los elementos que provee riqueza socio-emocional. Por ello, el éxito de la empresa depende en gran medida de la manera en que la familia se ha organizado y cómo gestiona su relación con la misma.

\section{Implicaciones}

La presente investigación tiene relevancia para propietarios, gerentes, consultores, así como para académicos que estudian la internacionalización de la empresa familiar y los factores que la motivan. Los propietarios, gerentes y consultores de empresas familiares deben tener presente que la cultura familiar permite la convergencia de los sistemas familiar y empresarial, facilitando la combinación única e interacción de recursos y capacidades para lograr crear ventajas competitivas y diferenciarse en su desempeño, tanto si las metas empresariales son económicas o no, como puede ser el cuidar y preservar los lazos familiares. El compromiso de la familia con la empresa desarrolla su cultura organizacional y este constituye un importante recurso estratégico que la empresa puede utilizar para desarrollar ventajas competitivas difícilmente imitables, orientándola predominantemente hacia su mercado doméstico, resultando en una menor propensión a la internacionalización y constituye uno de los elementos que 
proveen vinculación socio-emocional a los miembros de la familia que participan en su administración.

En el mundo académico, la presente investigación aporta conocimiento sobre internacionalización de la empresa familiar. En Ecuador no se han identificado estudios de esta naturaleza, por lo cual se busca contribuir con evidencia empírica para el país. Es importante se incorpore en los programas de estudio referentes a la internacionalización empresarial, la variable "familia" como un mecanismo que apoye el emprendimiento y desarrollo de la empresa familiar, misma que representa la forma más extendida de negocio en el mundo, y constituye el mayor número de empresas, con gran relevancia en el contexto económico de las naciones.

\section{Recomendaciones}

Los resultados obtenidos pueden ser aplicados de manera práctica en la gestión de la mediana empresa familiar ecuatoriana, mismos que son consistentes con la literatura y orientarán a la empresa en sus objetivos de crecimiento, desarrollo y sostenibilidad. La presente investigación considera el sector florícola y agro industrial. Se recomienda profundizar e incorporar a otros sectores económicos, considerando la posibilidad de incluir sectores exportadores tanto de bienes como de servicios, determinando si los factores de internacionalización considerados en el presente estudio tienen el mismo comportamiento. Se recomienda que futuros estudios analicen cómo la naturaleza y carácter familiar de empresa es desafiado por la internacionalización, considerando las diferencias en su proceso de internacionalización y cómo éste se desarrolla comparando la empresa familiar con la no familiar.

\section{Bibliografía}

Astrachan, J. H., Klein, S. B., \& Smyrnios, K. X. (2002). The F-PEC scale of family influence: A proposal for solving the family business definition problem. Family Business Review, 15(1), 45-58. doi: 10.1111/j.1741-6248.2002.00045.x

Berent-Braun, M. M., \& Uhlaner, L. M. (2010). Family governance practices and teambuilding: paradox of the enterprising family. Journal of Small Business Economics, 38(1), 103-119. doi: $10.1007 / \mathrm{s} 11187-010-9269-4$

Calabrò, A., \& Mussolino, D. (2013). How do boards of directors contribute to family SME export intensity? The role of formal and informal governance mechanisms. Journal of Management \& Governance, 17(2), 363-403. doi: 10.1007/s10997-011-9180-7

Cerrato, D., \& Piva, M. (2012). The internationalization of small and medium-sized enterprises: the effect of family management, human capital and foreign ownership. Journal of Management \& Governance, 16(4), 617-644. doi: 10.1007/s10997-010-9166-x

Comunidad Andina. (2008). Decisión 702. Sistema Andino de Estadísticas de la PYME. Lima-Perú: Secretaría General de la Comunidad Andina. Recuperado de http://www.comunidadandina.org/Seccion. aspx?id=79\&tipo=TE\&title=pymes.

Comunidad Andina. (2009). Resolución 1260. Disposición Técnica para la Transmisión de Datos de Estadística de PYME de los Países Miembros de la Comunidad Andina. Lima-Perú: Secretaría General de la Comunidad Andina. Recuperado de http://www.comunidadandina.org/Seccion.aspx?id=79\&tipo=TE\&title=pymes.

Craig, J. B., Dibrell, C., \& Garrett, R. (2014). Examining relationships among family influence, family culture, flexible planning systems, innovativeness and firm performance. Journal of Family Business Strategy, 5(3), 229-238. doi: 10.1016/j.jfbs.2013.09.002 
Eddleston, K. A., Kellermanns, F. W., \& Zellweger, T. M. (2012). Exploring the entrepreneurial behavior of family firms: Does the Stewardship Perspective explain differences? Entrepreneurship Theory and Practice, 36(2), 347-367. doi: 10.1111/j.1540-6520.2010.00402.x

Ensley, M. D., \& Pearson, A. W. (2005). An exploratory comparison of the behavioral dynamics of top management teams in family and nonfamily new ventures: Cohesion, conflict, potency and consensus. Entrepreneurship Theory and Practice, 29(3), 267-284. doi: 10.1111/j.15406520.2005.00082.X

Fernández, Z., \& Nieto, M. J. (2005). Internationalization strategy of small and medium-sized family businesses: Some influential factors. Family Business Review, 18(1), 77-89. doi: 10.1111/j.17416248.2005.00031.x

Graves, C., \& Thomas, J. (2006). Internationalization of australian family businesses: A managerial capabilities perspective. Family Business Review, 19(3), 207-224. doi: 10.1111/j.17416248.2006.00066.x

Graves, C., \& Thomas, J. (2008). Determinants of the internationalization pathways of family firms: An examination of family influence. Family Business Review, 21(2), 151-167. doi: 10.1111/j.17416248.2008.00119.x

Hair, J. F., Ringle, C. M., \& Sarstedt, M. (2011). PLS-SEM: Indeed a Silver Bullet. The Journal of Marketing Theory and Practice, 19(2), 139-152. doi: 10.2753/mtp1069-6679190202

Hair, J. F., Ringle, C. M., \& Sarstedt, M. (2013). Partial Least Squares Structural Equation Modeling: Rigorous Applications, Better Results and Higher Acceptance. Long Range Planning, 46(1-2), 112. doi: 10.1016/j.lrp.2013.01.001

Hair, J. F., Sarstedt, M., Pieper, T. M., \& Ringle, C. M. (2012). The Use of Partial Least Squares Structural Hiebl, M. R. W. (2013). Management accounting in the family business: tipping the balance for survival. Journal of Business Strategy, 34(6), 19-25. doi: 10.1108/jbs-07-2013-0052

INEC. (2013). Directorio de Empresas 2013 [Empresas_2013.sav]. Recuperado de http://www.ecuadorencifras.gob.ec/directoriodeempresas/

INEC. (2014). Compendio Estadístico 2014. Quito-Ecuador: Instituto Nacional de Estadística y Censos. Recuperado de http://www.ecuadorencifras.gob.ec/directoriodeempresas/.

Klein, S. B., Astrachan, J. H., \& Smyrnios, K. X. (2005). The F-PEC scale of family influence: Construction, validation, and further implication for theory. Entrepreneurship Theory and Practice, 29(3), 321-339. doi: 10.1111/j.1540-6520.2005.00086.x

Kock, N. (2015). WarpPLS 5.0 User Manual. Laredo, TX: ScriptWarp System.

Kontinen, T., \& Ojala, A. (2012). Internationalization pathways among family-owned SMEs. International Marketing Review, 29(5), 496-518. doi: 10.1108/02651331211260359

Kuivalainen, O., Saarenketo, S., \& Puumalainen, K. (2012). Start-up patterns of internationalization: A framework and its application in the context of knowledge-intensive SMEs. European Management Journal, 30(4), 372-385. doi: 10.1016/j.emj.2012.01.001

Kuivalainen, O., Sundqvist, S., Saarenketo, S., \& McNaughton, R. (2012). Internationalization patterns of small and medium-sized enterprises. International Marketing Review, 29(5), 448-465. doi: $10.1108 / 02651331211260331$

Lind, D. A., Marchal, W. G., \& Wathen, S. A. (2008). Estadística Aplicada a los Negocios y la Economía (15th ed ed.). México, México: McGraw-Hill.

Merino, F., Monreal-Pérez, J., \& Sánchez-Marín, G. (2012, 25-May-2012). Family firm internationalization: Influence of familiness on the Spanish firm export activity. Kiel Institute for the World Economy, 1-33. Recuperado de http://hdl.handle.net/10419/60329.

Miller, D., Le Breton-Miller, I., \& Scholnick, B. (2008). Stewardship vs. Stagnation: An empirical comparison of small family and non-family businesses. Journal of Management Studies, 45(1), 5178. doi: 10.1111/j.1467-6486.2007.00718.x

Mitter, C., Duller, C., Feldbauer-Durstmüller, B., \& Kraus, S. (2014). Internationalization of family firms: the effect of ownership and governance. Review of Managerial Science, 8(1), 1-28. doi: 10.1007/s11846-012-0093-X 
Naldi, L., Nordqvist, M., Sjoberg, K., \& Wiklund, J. (2007). Entrepreneurial orientation, risk taking, and performance in family firms. Family Business Review, 20(1), 33-47. doi: 10.1111/j.17416248.2007.00082.x

Nitzl, C., Roldan, J. L., \& Cepeda, G. (2016). Mediation analysis in partial least squares path modeling: Helping researchers discuss more sophisticated models. Industrial Management \& Data Systems, 116(9), 1849-1864. doi: 10.1108/imds-07-2015-0302

Pino, R. M. (2008). La relación entre el sector industrial y el tamaño de empresa con las prácticas de calidad total y el desempeño organizacional. (Tesis doctoral), CENTRUM Católica del Perú. Pontificia Universidad Católica del Perú, Lima, Perú. Recuperado de http://tesis.pucp.edu.pe/repositorio/handle/123456789/782

Riahi-Belkaoui, A. (1998). The effects of the degree of internationalization on firm performance. International Business Review, 7(3), 315-321. doi: 10.1016/S0969-5931(98)00013-4

Rutherford, M. W., Kuratko, D. F., \& Holt, D. T. (2008). Examining the link between "familiness" and performance: Can the F-PEC untangle the family Business Theory Jungle? Entrepreneurship Theory and Practice, 32(6), 1089-1109. doi: 10.1111/j.1540-6520.2008.00275.x

Salomón, J. O. (2010). Capacidades gerenciales en las empresas familiares peruanas: Un estudio descriptivo. (Tesis doctoral), CENTRUM Católica del Perú. Pontificia Universidad Católica del Perú, Lima, Perú. Recuperado de http://tesis.pucp.edu.pe/repositorio/handle/123456789/1605

Sarstedt, M., Ringle, C. M., \& Hair, J. F. (2014). PLS-SEM: Looking Back and Moving Forward. Long

Sciascia, S., Mazzola, P., Astrachan, J. H., \& Pieper, T. M. (2012). The role of family ownership in international entrepreneurship: exploring nonlinear effects. Small Business Economics, 38(1), 1531. doi: $10.1007 / \mathrm{s} 11187-010-9264-9$

Segaro, E. (2012). Internationalization of family SMEs: the impact of ownership, governance, and top management team. Journal of Management \& Governance, 16(1), 147-169. doi: 10.1007/s10997010-9145-2

Segaro, E., Larimo, J., \& Jones, M. (2014). Internationalisation of family small and medium sized enterprises: The role of stewardship orientation, family commitment culture and top management team. International Business Review, 23(2), 381-395. doi: 10.1016/j.ibusrev.2013.06.004

Smith, D., Hair, J. F., \& Ferguson, K. (2014). An investigation of the effect of family influence on Commitment-Trust in retailer-vendor strategic partnerships. Journal of Family Business Strategy, 5(3), 252-263. doi: 10.1016/j.jfbs.2013.11.005

Swoboda, B., \& Olejnik, E. (2013). A taxonomy of small- and medium-sized international family firms. Journal of International Entrepreneurship, 11(2), 130-157. doi: 10.1007/s10843-012-0101-x

Zahra, S. A. (2012). Organizational learning and entrepreneurship in family firms: exploring the moderating effect of ownership and cohesion. Small Business Economics, 38(1), 51-65. doi: 10.1007/s11187010-9266-7

Zahra, S. A., Hayton, J. C., Neubaum, D. O., Dibrell, C., \& Craig, J. (2008). Culture of family commitment and strategic flexibility: The moderating effect of stewardship. Entrepreneurship Theory and Practice, 32(6), 1035-1054. doi: 10.1111/j.1540-6520.2008.00271.x

\section{Apéndice A: Encuesta Aplicada y Resultados Obtenidos}

Tabla A1. Preguntas y Resultado Obtenido para la Variable Cultura Familiar

\begin{tabular}{|c|c|c|c|c|c|c|c|c|c|c|c|c|}
\hline \multicolumn{13}{|c|}{$\begin{array}{l}\text { En qué medida está usted de acuerdo con las siguientes ideas o afirmaciones. 1: No, en absoluto; } 5 \text { : } \\
\text { Totalmente, en gran medida }\end{array}$} \\
\hline \multirow{2}{*}{$\begin{array}{c}\text { Pregunta / } \\
\text { Cultura } \\
\text { Familiar/Variabl } \\
\mathrm{e}\end{array}$} & \multicolumn{2}{|c|}{1} & \multicolumn{2}{|c|}{2} & \multicolumn{2}{|c|}{3} & \multicolumn{2}{|c|}{4} & \multicolumn{2}{|c|}{5} & $\begin{array}{c}\text { Total } \\
\text { de } \\
\text { casos }\end{array}$ & $\begin{array}{c}\text { Casos } \\
\text { perdido } \\
\mathrm{s}\end{array}$ \\
\hline & $\begin{array}{c}\text { Frec } \\
\text { a }\end{array}$ & $\begin{array}{c}\text { Porc } \\
b\end{array}$ & $\begin{array}{c}\text { Fre } \\
c\end{array}$ & $\begin{array}{c}\text { Por } \\
\mathrm{c}\end{array}$ & $\begin{array}{c}\text { Fre } \\
\mathrm{c}\end{array}$ & $\begin{array}{c}\text { Por } \\
\mathrm{c}\end{array}$ & $\begin{array}{c}\text { Fre } \\
\mathrm{c}\end{array}$ & $\begin{array}{c}\text { Por } \\
\mathrm{c}\end{array}$ & $\begin{array}{c}\text { Fre } \\
\mathrm{c}\end{array}$ & $\begin{array}{c}\text { Por } \\
\text { c }\end{array}$ & $\begin{array}{c}\text { válido } \\
\mathrm{s}\end{array}$ & \\
\hline
\end{tabular}




\begin{tabular}{|c|c|c|c|c|c|c|c|c|c|c|c|c|c|c|}
\hline (a) & $\begin{array}{l}\text { Los } \\
\text { miembros de } \\
\text { la familia } \\
\text { comparten } \\
\text { valores } \\
\text { similares }\end{array}$ & $\begin{array}{c}\mathrm{FC} \\
1\end{array}$ & 3 & 2.6 & 1 & .9 & 14 & $\begin{array}{c}12 . \\
0\end{array}$ & 19 & $\begin{array}{c}16 . \\
2\end{array}$ & 80 & $\begin{array}{c}68 . \\
4\end{array}$ & 117 & \\
\hline (b) & $\begin{array}{l}\text { La familia y } \\
\text { la empresa } \\
\text { comparte } \\
\text { valores } \\
\text { similares }\end{array}$ & $\begin{array}{c}\mathrm{FC} \\
2\end{array}$ & & & & & 2 & 1.7 & 40 & $\begin{array}{c}34 . \\
2\end{array}$ & 73 & $\begin{array}{c}62 . \\
4\end{array}$ & 115 & 2 \\
\hline (c) & $\begin{array}{l}\text { Apoyamos el } \\
\text { negocio } \\
\text { familiar en } \\
\text { conversacion } \\
\text { es con } \\
\text { amigos, } \\
\text { empleados y } \\
\text { otros } \\
\text { miembros de } \\
\text { la familiar }\end{array}$ & $\begin{array}{c}\mathrm{FC} \\
3\end{array}$ & & & 4 & 3.4 & 7 & 6.0 & 13 & $\begin{array}{c}11 . \\
1\end{array}$ & 93 & $\begin{array}{c}79 . \\
5\end{array}$ & 117 & \\
\hline (d) & $\begin{array}{l}\text { Los } \\
\text { miembros de } \\
\text { la familia } \\
\text { están } \\
\text { dispuestos a } \\
\text { esforzarse } \\
\text { más allá de } \\
\text { lo que } \\
\text { normalment } \\
\text { e se espera } \\
\text { para lograr } \\
\text { que la } \\
\text { empresa } \\
\text { familiar sea } \\
\text { exitosa }\end{array}$ & $\begin{array}{c}\mathrm{FC} \\
4\end{array}$ & & & & & & & 15 & $\begin{array}{c}12 . \\
8\end{array}$ & 102 & $\begin{array}{c}87 . \\
2\end{array}$ & 117 & \\
\hline (e) & $\begin{array}{l}\text { Sentimos } \\
\text { lealtad con } \\
\text { la empresa } \\
\text { familiar }\end{array}$ & $\begin{array}{c}\mathrm{FC} \\
5\end{array}$ & & & & & 8 & 6.8 & & & 109 & $\begin{array}{c}93 . \\
2\end{array}$ & 117 & \\
\hline (f) & $\begin{array}{l}\text { Estamos } \\
\text { orgullosos de } \\
\text { contarles a } \\
\text { otros que } \\
\text { somos parte } \\
\text { de un } \\
\text { negocio } \\
\text { familiar }\end{array}$ & $\begin{array}{c}\mathrm{FC} \\
6\end{array}$ & & & & & 7 & 6.0 & 6 & 5.1 & 104 & $\begin{array}{c}88 . \\
9\end{array}$ & 117 & \\
\hline (g) & $\begin{array}{l}\text { Se pueden } \\
\text { conseguir } \\
\text { numerosos } \\
\text { logros en el } \\
\text { largo plazo } \\
\text { al participar } \\
\text { en la }\end{array}$ & $\begin{array}{c}\mathrm{FC} \\
7\end{array}$ & & & & & 4 & 3.4 & 13 & $\begin{array}{c}11 . \\
1\end{array}$ & 100 & $\begin{array}{c}85 . \\
5\end{array}$ & 117 & \\
\hline
\end{tabular}


empresa

familiar

\begin{tabular}{|c|c|c|c|c|c|c|c|c|c|c|}
\hline (h) & $\begin{array}{l}\text { Estamos de } \\
\text { acuerdo con } \\
\text { los objetivos, } \\
\text { planes y } \\
\text { políticas de } \\
\text { la empresa } \\
\text { familiar }\end{array}$ & $\begin{array}{c}\mathrm{FC} \\
8\end{array}$ & 7 & 6.0 & 31 & $\begin{array}{c}26 . \\
5\end{array}$ & 77 & $\begin{array}{c}65 . \\
8\end{array}$ & 115 & 2 \\
\hline (i) & $\begin{array}{l}\text { Realmente } \\
\text { nos interesa } \\
\text { el futuro de } \\
\text { la empresa } \\
\text { familiar }\end{array}$ & $\begin{array}{c}\mathrm{FC} \\
9\end{array}$ & & & 7 & 6.0 & 109 & $\begin{array}{c}93 . \\
2\end{array}$ & 116 & 1 \\
\hline
\end{tabular}

Tabla A2. Resultado Obtenido para el indicador Intensidad Exportadora

De las ventas totales: ¿Qué porcentaje corresponde a ventas en mercados internacionales?

\begin{tabular}{c|c|r|r|r|r|}
$\begin{array}{c}\text { Porcentaje de ventas en mercados } \\
\text { internacionales }\end{array}$ & Frecuencia & Porcentaje & $\begin{array}{c}\text { Porcentaje } \\
\text { válido }\end{array}$ & \multicolumn{2}{|c|}{$\begin{array}{c}\text { Porcentaje } \\
\text { acumulado }\end{array}$} \\
\hline Válidos (FSTS) & .10 & 2 & 1.7 & 1.7 & 1.7 \\
\hline & .20 & 1 & .9 & .9 & 4.3 \\
\hline & .30 & 2 & 1.7 & 1.7 & 6.8 \\
\hline & .40 & 3 & 2.6 & 2.6 & 10.3 \\
\hline & .50 & 4 & 3.4 & 3.4 & 12.8 \\
\hline & .60 & 3 & 2.6 & 2.6 & 21.4 \\
\hline & .70 & 10 & 8.5 & 8.5 & 41.9 \\
\hline & .80 & 5 & 4.3 & 4.3 & 100.0 \\
\hline & .90 & 19 & 16.2 & 16.2 & \\
\hline
\end{tabular}

\section{Apéndice B: Resultados Estadísticos}

Tabla B1. Estadístico de Observaciones

\begin{tabular}{|c|c|c|c|c|c|c|c|c|c|c|}
\hline & & & & & & \multicolumn{2}{|c|}{ Asimetría } & \multicolumn{2}{|c|}{ Curtosis } & \multirow{2}{*}{$\frac{\text { \% Datos }}{\text { perdidos }}$} \\
\hline & $\mathrm{N}$ & Mín & Máx & Media & $\begin{array}{l}\text { Desv. } \\
\text { típ }\end{array}$ & Estadístico & $\begin{array}{l}\text { Error } \\
\text { típ. }\end{array}$ & Estadístico & $\begin{array}{c}\text { Error } \\
\text { típ. }\end{array}$ & \\
\hline FC1 & 117 & 1 & 5 & 4.47 & .925 & -1.942 & .224 & 3.692 & .444 & \\
\hline FC2 & 115 & 3 & 5 & 4.62 & .523 & -.861 & .226 & -.475 & .447 & $1.71 \%$ \\
\hline FC3 & 117 & 2 & 5 & 4.67 & .743 & -2.326 & .224 & 4.675 & .444 & \\
\hline FC4 & 117 & 4 & 5 & 4.87 & .336 & -2.253 & .224 & 3.130 & .444 & \\
\hline FC5 & 117 & 3 & 5 & 4.86 & .507 & -3.465 & .224 & 10.179 & .444 & \\
\hline FC6 & 117 & 3 & 5 & 4.83 & .513 & -2.963 & .224 & 7.536 & .444 & \\
\hline FC7 & 117 & 3 & 5 & 4.82 & .466 & -2.658 & .224 & 6.503 & .444 & \\
\hline FC8 & 115 & 3 & 5 & 4.61 & .603 & -1.286 & .226 & .633 & .447 & $1.71 \%$ \\
\hline FC9 & 116 & 4 & 5 & 4.94 & .239 & -3.741 & .225 & 12.207 & .446 & $0.85 \%$ \\
\hline FSTS & 117 & .10 & 1.00 & .8726 & .21157 & -1.944 & .224 & 3.277 & .444 & \\
\hline $\begin{array}{l}\text { N } \\
\text { válido }\end{array}$ & 112 & & & & & & & & & $4.27 \%$ \\
\hline
\end{tabular}


Tabla B2. Prueba de Normalidad

\begin{tabular}{lcccccc}
\hline & \multicolumn{2}{c}{ Kolmogorov-Smirnov ${ }^{\text {a }}$} & \multicolumn{3}{c}{ Shapiro-Wilk } \\
\cline { 2 - 7 } & Estadístico & gl & Sig. & Estadístico & gl & Sig. \\
& & & & & & \\
\hline FC1 & .406 & 112 & .000 & .626 & 112 & .000 \\
\hline FC2 & .397 & 112 & .000 & .654 & 112 & .000 \\
\hline FC3 & .481 & 112 & .000 & .494 & 112 & .000 \\
\hline FC5 & .522 & 112 & .000 & .387 & 112 & .000 \\
\hline FC6 & .537 & 112 & .000 & .281 & 112 & .000 \\
\hline FC7 & .526 & 112 & .000 & .320 & 112 & .000 \\
\hline FC8 & .509 & 112 & .000 & .409 & 112 & .000 \\
\hline FC9 & .411 & 112 & .000 & .648 & 112 & .000 \\
\hline FSTS & .541 & 112 & .000 & .209 & 112 & .000 \\
\hline Nota ${ }^{*}$ a Corrección de la significación de Lilliefors. & .295 & 112 & .000 & .679 & 112 & .000 \\
\hline
\end{tabular}

Tabla B3. Comparación de Medias por Sector

\begin{tabular}{lcrrr} 
& $\begin{array}{c}\text { U de Mann- } \\
\text { Whitney }\end{array}$ & $\begin{array}{c}\text { W de } \\
\text { Wilcoxon }\end{array}$ & Z & \multicolumn{2}{c}{$\begin{array}{c}\text { Sig. asintót. } \\
\text { (bilateral) }\end{array}$} \\
\hline FC1 & 1425.000 & 4275.000 & -1.038 & .299 \\
\hline FC2 & 1350.000 & 4051.000 & -1.269 & .205 \\
\hline FC3 & 1419.000 & 4269.000 & -1.258 & .208 \\
\hline FC4 & 1494.000 & 4344.000 & -.795 & .427 \\
\hline FC5 & 1567.500 & 2470.500 & -.097 & .922 \\
\hline FC6 & 1473.500 & 4323.500 & -1.058 & .290 \\
\hline FC7 & 1500.500 & 4350.500 & -.692 & .489 \\
\hline FC8 & 1413.000 & 2316.000 & -.845 & .398 \\
\hline FC9 & 1452.000 & 4302.000 & -1.197 & .231 \\
\hline Nota. ${ }^{\text {a }}$ Variable de agrupación: SECTOR. & & \\
\hline
\end{tabular}

Tabla B4. Correlación entre Indicadores Reflectivos

\begin{tabular}{llllllllll}
\hline & FSTS & FC1 & FC2 & FC3 & FC4 & FC5 & FC6 & FC7 & FC9 \\
\hline FSTS & 1.000 & & & & & & & & \\
\hline FC1 & -0.150 & 1.000 & & & & & & & \\
\hline FC2 & -0.173 & 0.359 & 1.000 & & & & & & \\
\hline FC3 & -0.026 & 0.255 & 0.117 & 1.000 & & & & & \\
\hline FC4 & -0.110 & 0.196 & 0.379 & 0.311 & 1.000 & & & & \\
\hline FC5 & -0.083 & 0.212 & 0.324 & 0.473 & 0.605 & 1.000 & & & \\
\hline FC6 & -0.107 & 0.371 & 0.230 & 0.550 & 0.522 & 0.373 & 1.000 & & \\
\hline FC7 & -0.120 & 0.177 & 0.248 & 0.498 & 0.567 & 0.552 & 0.663 & 1.000 & \\
\hline FC9 & -0.120 & 0.088 & 0.161 & 0.568 & 0.334 & 0.502 & 0.620 & 0.600 & 1.000 \\
\hline
\end{tabular}

